Research Paper

\title{
The clinical characteristics and prognostic factors of combined Hepatocellular Carcinoma and Cholangio- carcinoma, Hepatocellular Carcinoma and Intrahepatic Cholangiocarcinoma after Surgical Resection: A propensity score matching analysis
}

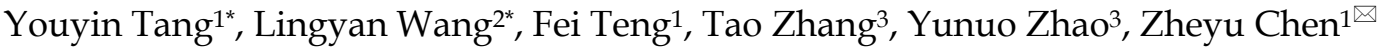 \\ 1. Department of Liver Surgery, Liver Transplantation Center, West China Hospital of Sichuan University, No. 37 GuoXue Alley, Chengdu 610041, P.R. China. \\ 2. Department of Obstetrics and Gynecology Nursing, Key Laboratory of Birth Defects and Related Disease of Women and Children, West China Second \\ University Hospital, Sichuan University, No. 20 South Renmin Road, Chengdu 610041, P.R. China. \\ 3. Department of Biotherapy, West China Hospital and State Key Laboratory of Biotherapy, Sichuan University, No. 37 GuoXue Alley, Chengdu 610041, P.R. \\ China. \\ *These authors contributed equally to this study. \\ $\triangle$ Corresponding author: Zheyu Chen, E-mail: chenzheyu@scu.edu.cn; Department of Liver Surgery, Liver Transplantation Center, West China Hospital of \\ Sichuan University, No. 37 GuoXue Alley, Chengdu 610041, P.R. China. Tel.: +86 18982030423. \\ (C) The author(s). This is an open access article distributed under the terms of the Creative Commons Attribution License (https://creativecommons.org/licenses/by/4.0/). \\ See http://ivyspring.com/terms for full terms and conditions.
}

Received: 2020.07.20; Accepted: 2020.10.13; Published: 2021.01.01

\begin{abstract}
Background: Clinical characteristics and prognosis among combined hepatocellular carcinoma (HCC) and cholangiocarcinoma (cHCC-CC) with HCC and intrahepatic cholangiocarcinoma (ICC) were inconsistent in previous studies. The aim of this study was to compare postoperative prognosis among cHCC-CC, HCC and ICC, and investigated the prognostic risk factor of cHCC-CC after surgical resection.

Methods: A total of 1041 eligible patients with pathological diagnosis of cHCC-CC $(n=135), \mathrm{HCC}$ $(n=698)$ and ICC $(n=208)$ were enrolled in this study. Univariate and multivariate Cox analysis were applied for assessing important risk factors. cHCC-CC were further 1:1 matched with HCC and ICC on important clinical risk factors. Survival curves of matched and unmatched cohorts were depicted by Kaplan-Meier method with log-rank test.

Results: Patients with $\mathrm{CHCC}-\mathrm{CC}$ had similar rate of sex, age and cirrhosis with HCC $(p<0.05)$ and comparable incidence of hepatitis B or C with ICC $(p=0.197)$. Patients of $c H C C-C C$ had intermediate prognosis between HCC and ICC, with median overall survival (OS) time of cHCC-CC, HCC and ICC of 20.5 months, 35.7 months and 11.6 months $(p<0.001)$. In matched cohorts, the OS of cHCC-CC were worse than HCC $(p<0.001)$ but comparable with ICC $(p=0.06)$, while the disease-free survival (DFS) of cHCC-CC was worse than HCC but better than ICC $(p<0.05)$. And lymph node infiltration and postoperative transarterial chemoembolization (TACE) were independent risk factors of $\mathrm{cHCC}-\mathrm{CC}$ associated with prognosis.

Conclusion: The long term survival of $\mathrm{CHCC}-\mathrm{CC}$ was worse than HCC but comparable with ICC when matched on albumin level, tumor size, lymph node infiltration, tumor stage and margin. Presence of lymph node infiltration and no postoperative TACE were associated with poor prognosis of $\mathrm{cHCC}-\mathrm{CC}$.
\end{abstract}

Key words: combined hepatocellular carcinoma and cholangiocarcinoma; surgical resection; clinical features; long-term survival; propensity score matching 


\section{Introduction}

Combined hepatocellular carcinoma (HCC) and cholangiocarcinoma (cHCC-CC) is a relatively rare type of liver tumor, with an incidence of $0.8 \%-6.5 \%$ in primary liver carcinoma [1-6]. Considering of this special phenotype of primary liver malignancy, several medical terms, such as mixed HCC and cholangiocarcinoma or dual-phenotype HCC, were applied to describe cHCC-CC for decades [7, 8]. In recent years, a valuable nomenclature by World Health Organization (WHO) defined cHCC-CC as a single nodule with both differentiation of hepatocellular carcinoma (HCC) and intrahepatic cholangiocarcinoma (ICC), and removed the subtype of cholangiolocellular carcinoma (CLC) which was considered as a subtype of cHCC-CC in the $4^{\text {th }}$ edition $[9,10]$.

Albeit the clinical characteristics and prognosis of among cHCC-CC, HCC and ICC had been widely discussed for years, the results yielded in their studies were still inconsistent [2, 4, 6, 11-14]. Most of the previous studies found that $\mathrm{CHCC}-\mathrm{CC}$ had worse prognosis than HCC [2, 4-6, 12, 15-19], while some of them suggest the prognosis of cHCC-CC was better than ICC $[2,4,6]$, and others insisted opposite conclusion [5, 12, 15-19]. In the literature review, large-scale studies regarding to the long-term outcome among CHCC-CC, HCC and ICC were limited and a recently western series which comprised 623 patients found that no significant difference of 5-year survival rate of CHCC-CC, HCC as well as ICC after surgical resection [11]. However, they only included 47 cHCC-CC patients. Thus, the conclusions yielded in their studies were still limited, meaning the clinical characteristics and prognosis of cHCC-CC compared to HCC or ICC were still inconclusive.

In the current study, we aimed to compare the clinical features and long term survival of cHCC-CC compared with HCC and ICC after surgical resection, identifying survival risk factors of cHCC-CC after surgical resection.

\section{Materials and Methods}

\section{Patient selection}

This study was approved by the Ethics Committee of West China Hospital of Sichuan University. And inform consent was waived because no individual information was disclosed in this study. The medical records of patients diagnosed with cHCC-CC, HCC and ICC during January 2012 to June 2017 at West China Hospital were retrospectively reviewed. The diagnosis of CHCC-CC abided by
World Health Organization classification of digestive system tumours, and cholangiolocellular carcinoma (CLC) were excluded in this study $[9,10]$. HCC with expression of keratin (K) 19 was also excluded since the distinction of K19 positive HCC and CHCC-CC was ambiguous [20].

The additional inclusion and exclusion criteria were: Inclusion criteria: i) patients had complete medical records and follow-up; ii) patients had pathological diagnosis of CHCC-CC, ICC and K19 negative HCC according to the latest $\mathrm{WHO}$ classification; Exclusion criteria: i) patients only received exploratory surgeries rather than surgical resection; ii) patients had distant metastasis (M1 stage); iii) patients had co-malignancies in other organs. Figure 1 was the patient selection flow chart.

\section{Study design and propensity score matching}

In order to minimize the impact of distinguishing clinical characteristics among cHCCCC, HCC and ICC patients, patients were further enrolled into two separate matched cohorts: i) cHCCCC were 1:1 matched with HCC on albmin (ALB) level, tumor size, lymph node (LN) infiltration, $8^{\text {th }}$ American Joint Committee on Cancer (AJCC) staging and margin; ii) CHCC-CC were 1:1 matched with ICC regarding to ALB level, tumor size, lymph node infiltration, $8^{\text {th }}$ AJCC staging and margin.

\section{Preoperative management and surgery}

All patients' baseline characteristic and surgical details as well as pathological features were carefully reviewed. Enhanced imaging examination including ultrasonography, computed tomography, magnetic resonance imaging were used to preoperatively assess the tumor size, location, invasion of portal vein, bile duct or hepatic arteries and presence of intro-hepatic or extra-hepatic metastases in all patients. Patients with Child-Pugh class A liver function and performance status score less than 2 were eligible for surgical resection. Surgical methods were assessed by preoperative multidisciplinary team discussion. In the present study, hypersplenia was diagnosed by: i) enlarged spleen (thickness of spleen was more than $4 \mathrm{~cm}$ in ultrasonography); ii) peripheral cytopenias, including the decreasing of red blood cells, white blood cells and platelets. Major liver resection was defined as more than two segments resection while the minor liver resection was considered as $\leq 2$ segments resection. All enrolled patients had routine follow-up at first month and every 6 month subsequently until May 2020. The primary endpoint of this study was overall survival (OS) and OS was calculated from the time receiving surgery to the time of death or May 2020. The second end point was 
disease-free survival (DFS) which was defined as the duration between surgical resection and recurrence or metastasis.

\section{Statistical analysis}

Power analysis was performed to ensure sufficient subjects in this study. An estimated 695 patients would be needed to provide $90 \%$ power for 5 -year overall survival log-rank test with a two-sided a of 0.05 (Supplement Table 3). To compare baseline demographic and clinicopathologic characteristics among patients diagnosed with $\mathrm{CHCC}-\mathrm{CC}, \mathrm{HCC}$ and ICC, Kruskal-Wallis test (for continuous variables) as well as $\chi^{2}$ test and Fisher exact tests (for categorical variables) were all used in this study. Within the unmatched cohort and matched cohorts, survival curves were depicted using Kaplan-Meier methods with comparison of log-rank test. Univariate analysis and multivariate Cox regression analysis with step-wise selection were used to compare statistical difference of overall survival in unmatched and matched cohort. Variables with $p$ value $<0.1$ in univariate analysis were further selected in multivariate Cox regression analysis. A $p$-value $<0.05$ was considered statistically significant. All statistical analyses were conducted with EmpowerStats software, version 2.20.

\section{Results}

\section{Patient characteristics}

A total of 1041 patients were included in our study, with 135 patients in cHCC-CC group, 698 in HCC group and 208 in ICC group. Among them, there were 847 males, accounting for $81.4 \%$ of the whole cohort. In addition, 776 patients $(74.5 \%)$ were diagnosed at age $<60$ years. Surgical resection was applied for most patients $(98.3 \%)$, while anatomy resection was performed in 467 cases. Patients' baseline characteristics and pathological details were summarized in Table 1.

cHCC-CC patients tended to have more similar baseline clinical features with HCC, such as hypertension, diabetes mellitus, alanine aminotransferase (ALT) level, aspartate aminotransferase (AST) level, ALB level, total bilirubin level. The rate of liver cirrhosis in cHCC-CC patients was comparable with HCC (cHCC-CC versus HCC, $40.7 \%$ versus $51.0 \%$, $p=0.32$ ), yet higher than that in ICC patients (cHCC-CC vs. ICC, $50.4 \%$ vs. $43.3 \%, p<0.01$ ). The incidence of hepatitis B or C of cHCC-CC was similar with ICC (cHCC-CC vs. ICC, $50.4 \%$ vs. $43.3 \%$, $p=0.197$ ), but significantly lower than that of HCC (cHCC-CC vs. HCC, $50.4 \%$ vs. $73.9 \%, p<0.001$ ). Considering of collinearity between vascular invasion and tumor thrombus, we only included the tumor thrombus data of each patient, and micro vascular thrombus was also included as tumor thrombus [21]. And we found that the incidence of tumor thrombus of cHCC-CC $(37.0 \%)$ was higher than ICC (21.6\%) but less than HCC (51.1\%). And this was compared to previous study, which reported the incidence of vascular invasion was about $9 \%$ to $89.5 \%$ in cHCC-CC $[4,16,22]$.

Table 1. Patients' baseline characteristics of $\mathrm{CHCC}-\mathrm{CC}, \mathrm{HCC}$ and ICC in the whole cohort prior to match

\begin{tabular}{|c|c|c|c|c|}
\hline Variable & $\begin{array}{l}\text { cHCC-CC } \\
(\mathrm{n}=135)\end{array}$ & $\begin{array}{l}\text { HCC } \\
(n=698)\end{array}$ & $\operatorname{ICC}(n=208)$ & $p$-value \\
\hline Sex, male, n (\%) & & & & $<0.001$ \\
\hline Male & $118(87.4)$ & $590(84.5)$ & $139(66.8)$ & \\
\hline Female & $17(12.6)$ & $108(15.5)$ & $69(33.2)$ & \\
\hline Age, year & & & & $<0.001$ \\
\hline$\leq 60$ & $105(77.8)$ & $547(78.4)$ & $124(59.6)$ & \\
\hline$>60$ & $30(22.2)$ & $151(21.6)$ & $84(40.4)$ & \\
\hline Hypertension, n (\%) & $13(9.6)$ & $65(9.3)$ & $35(16.8)$ & 0.008 \\
\hline Diabetes mellitus, $\mathrm{n}(\%)$ & $9(6.7)$ & $47(6.7)$ & $17(8.2)$ & 0.76 \\
\hline Hepatitis B/C, n (\%) & & & & $<0.001$ \\
\hline Present & $68(50.4)$ & $516(73.9)$ & $90(43.3)$ & \\
\hline Absent & 67 (49.6) & $182(26.1)$ & $118(56.7)$ & \\
\hline Hypersplenia, n (\%) & $15(11.1)$ & $54(7.7)$ & $8(3.8)$ & 0.036 \\
\hline $\mathrm{ALT}, \mathrm{U} / \mathrm{L}$, mean $\pm \mathrm{SD}$ & $53.6 \pm 94.2$ & $55.8 \pm 65.5$ & $41.6 \pm 50.2$ & 0.032 \\
\hline $\mathrm{AST}, \mathrm{U} / \mathrm{L}$, mean $\pm \mathrm{SD}$ & $60.3 \pm 129.4$ & $62.2 \pm 75.3$ & $45.4 \pm 48.5$ & 0.032 \\
\hline $\mathrm{ALB}, \mathrm{g} / \mathrm{L}$, mean $\pm \mathrm{SD}$ & $41.9 \pm 4.7$ & $40.3 \pm 6.6$ & $39.6 \pm 9.4$ & 0.011 \\
\hline $\mathrm{TB}, \mathrm{umol} / \mathrm{L}$, mean $\pm \mathrm{SD}$ & $15.8 \pm 9.8$ & $16.1 \pm 12.7$ & $16.5 \pm 18.3$ & 0.90 \\
\hline $\mathrm{PT}, \mathrm{s}$, mean $\pm \mathrm{SD}$ & $12.0 \pm 1.9$ & $12.5 \pm 1.3$ & $12.0 \pm 1.4$ & $<0.001$ \\
\hline $\mathrm{INR}$, mean $\pm \mathrm{SD}$ & $1.0 \pm 0.2$ & $1.1 \pm 0.1$ & $1.0 \pm 0.1$ & $<0.001$ \\
\hline $\mathrm{AFP}, \mathrm{ng} / \mathrm{ml}$, mean $\pm \mathrm{SD}$ & $286.6 \pm 476.0$ & $\begin{array}{l}449.5 \pm \\
533.5\end{array}$ & $178.5 \pm 407.5$ & $<0.001$ \\
\hline CA19-9, U/ml, mean \pm SD & $91.7 \pm 223.1$ & $32.6 \pm 68.7$ & $290.2 \pm 391.9$ & $<0.001$ \\
\hline $\begin{array}{l}\mathrm{CA} 125, \mathrm{U} / \mathrm{ml}, \\
\text { mean } \pm \mathrm{SD}\end{array}$ & $84.6 \pm 523.3$ & $35.3 \pm 221.8$ & $159.8 \pm 578.9$ & 0.011 \\
\hline $\mathrm{CEA}, \mathrm{ng} / \mathrm{ml}$, mean $\pm \mathrm{SD}$ & $5.8 \pm 28.1$ & $3.1 \pm 8.5$ & $22.0 \pm 97.9$ & $<0.001$ \\
\hline Liver fibrosis, n (\%) & & & & $<0.001$ \\
\hline No significant fibrosis & $19(14.1)$ & $19(3.1)$ & $39(18.8)$ & \\
\hline Significant fibrosis & $19(14.1)$ & $211(33.9)$ & $37(17.8)$ & \\
\hline Advanced fibrosis & $42(31.1)$ & $75(12.1)$ & 97 (46.6) & \\
\hline Liver cirrhosis & $55(40.7)$ & $317(51.0)$ & $35(16.8)$ & \\
\hline Tumor size, $\mathbf{n}(\%)$ & & & & 0.55 \\
\hline$\leq 5 \mathrm{~cm}$ & 44 (32.6) & $195(27.9)$ & $57(28.5)$ & \\
\hline$>5 \mathrm{~cm}$ & $91(67.4)$ & $503(72.1)$ & $143(71.5)$ & \\
\hline Tumor number, n (\%) & & & & $<0.001$ \\
\hline Single & $59(43.7)$ & $499(71.5)$ & $143(68.8)$ & \\
\hline Multiple & $76(56.3)$ & $199(28.5)$ & 65 (31.2) & \\
\hline Satellite lesions, n (\%) & $46(34.1)$ & $150(21.5)$ & $42(20.2)$ & 0.004 \\
\hline Tumor capsule, n (\%) & $23(17.0)$ & $116(16.6)$ & $82(39.4)$ & $<0.001$ \\
\hline Tumor thrombus, n (\%) & $50(37.0)$ & $357(51.1)$ & 45 (21.6) & $<0.001$ \\
\hline Lymph node infiltration, $\mathrm{n}(\%)$ & $18(13.3)$ & $25(3.6)$ & $31(14.9)$ & $<0.001$ \\
\hline Differentiation, n (\%) & & & & $<0.001$ \\
\hline Well & $5(3.7)$ & $7(1.1)$ & $5(2.4)$ & \\
\hline Moderate & $58(43.0)$ & $291(47.4)$ & $59(28.4)$ & \\
\hline Poor & $61(45.2)$ & $313(51.0)$ & $135(64.9)$ & \\
\hline Undifferentiated & $11(8.1)$ & $3(0.5)$ & $9(4.3)$ & \\
\hline $8^{\text {th }}$ AJCC stage, $n(\%)$ & & & & $<0.001$ \\
\hline I & $11(8.1)$ & $275(39.4)$ & $76(36.5)$ & \\
\hline II & $32(23.7)$ & $69(9.9)$ & $36(17.3)$ & \\
\hline III & $74(54.8)$ & $338(48.4)$ & $65(31.2)$ & \\
\hline IV & $18(13.3)$ & $16(2.3)$ & $31(14.9)$ & \\
\hline T stage, n (\%) & & & & $<0.001$ \\
\hline $\mathrm{T} 1$ & $15(11.1)$ & $279(40.0)$ & $85(40.9)$ & \\
\hline $\mathrm{T} 2$ & $35(25.9)$ & $69(9.9)$ & $39(18.8)$ & \\
\hline $\mathrm{T} 3$ & $52(38.5)$ & $64(9.2)$ & 45 (21.6) & \\
\hline $\mathrm{T} 4$ & $33(24.4)$ & $286(41.0)$ & $39(18.8)$ & \\
\hline N stage, $\mathbf{n}(\%)$ & & & & $<0.001$ \\
\hline No & $117(86.7)$ & $673(96.4)$ & $177(85.1)$ & \\
\hline N1 & 18 (13.3) & $25(3.6)$ & 31 (14.9) & \\
\hline
\end{tabular}




\begin{tabular}{|c|c|c|c|c|}
\hline Variable & $\begin{array}{l}\text { cHCC-CC } \\
(n=135)\end{array}$ & $\begin{array}{l}\text { HCC } \\
(n=698)\end{array}$ & ICC (n=208) & $p$-value \\
\hline M stage, n (\%) & & & & NA \\
\hline M0 & $135(100.0)$ & $698(100.0)$ & $208(100.0)$ & \\
\hline Transfusion & & & & 0.95 \\
\hline Yes & $18(13.3)$ & $95(14.0)$ & $27(13.2)$ & \\
\hline No & $117(86.7)$ & $586(86.0)$ & $178(86.8)$ & \\
\hline Blood loss, $\leq 400 \mathrm{ml}, \mathrm{n}(\%)$ & $80(59.3)$ & $469(68.9)$ & $148(72.2)$ & 0.036 \\
\hline ASA, n (\%) & 135 & 698 & 208 & 0.76 \\
\hline 1 & $1(2.1)$ & $11(3.5)$ & $3(2.6)$ & \\
\hline 2 & $43(89.6)$ & $242(77.1)$ & $88(77.2)$ & \\
\hline 3 & $4(8.3)$ & $56(17.8)$ & $22(19.3)$ & \\
\hline 4 & $0(0.0)$ & $4(1.3)$ & $1(0.9)$ & \\
\hline NA & $87(64.4)$ & $385(55.2)$ & $95(45.7)$ & \\
\hline Margin, n (\%) & & & & $<0.001$ \\
\hline R0 & $112(83.0)$ & $603(90.3)$ & $139(79.4)$ & \\
\hline R1 & $23(17.0)$ & $65(9.7)$ & $36(20.6)$ & \\
\hline Surgical method, n (\%) & & & & $<0.01$ \\
\hline Major resection & $61(45.2)$ & $339(48.6)$ & $137(65.9)$ & \\
\hline Minor resection & $64(47.4)$ & $287(41.1)$ & $50(24.0)$ & \\
\hline Resection+ Ablation & $7(5.2)$ & $60(8.6)$ & $18(8.7)$ & \\
\hline Liver transplantation & $3(2.2)$ & $12(1.7)$ & $3(1.4)$ & \\
\hline Anatomy resection, n (\%) & $57(43.8)$ & $271(40.9)$ & $139(71.3)$ & $<0.001$ \\
\hline Postoperative TACE, n (\%) & & & & $<0.001$ \\
\hline Yes & $40(29.6)$ & $326(46.7)$ & $80(38.5)$ & \\
\hline No & $95(70.4)$ & $372(53.3)$ & $128(61.5)$ & \\
\hline $\begin{array}{l}\text { Disease-free survival, } \mathrm{m} \text {, } \\
\text { mean } \pm \mathrm{SD}\end{array}$ & $28.9 \pm 28.2$ & $21.8 \pm 17.1$ & $12.1 \pm 16.0$ & $<0.001$ \\
\hline $\begin{array}{l}\text { Overall survival, } \\
\mathrm{m}, \text { mean } \pm \mathrm{SD}\end{array}$ & $32.3 \pm 27.4$ & $28.2 \pm 18.5$ & $15.7 \pm 16.0$ & $<0.001$ \\
\hline \multicolumn{5}{|c|}{$\begin{array}{l}\text { Abbreviation: cHCC-CC: Combined hepatocellular carcinoma and } \\
\text { cholangiocarcinoma; HCC: hepatocellular carcinoma; ICC: intrahepatic } \\
\text { cholangiocarcinoma; ALT: alanine aminotransferase; SD: standard deviation; AST: } \\
\text { aspartate aminotransferase; ALB: albumin; TB: total bilirubin; PT: prothrombin } \\
\text { time; INR: International Normalized Ratio; AFP: alpha fetoprotein; CEA: } \\
\text { carcinoembryonic antigen; AJCC: American Joint Committee on Cancer; ASA: } \\
\text { American Society of Anesthesiology; NA: not applicable; TACE: transhepatic } \\
\text { arterial chemotherapy and embolization; ref: reference. }\end{array}$} \\
\hline
\end{tabular}

cHCC-CC had higher rate of advanced tumor stage (AJCC stage III+IV) than HCC and ICC $(68.1 \%$ in $\mathrm{CHCC}-\mathrm{CC}$ vs. $50.7 \%$ in HCC and $46.1 \%$ in ICC, $p<0.001)$. ICC patients had higher rate of advanced tumor grade (poor or undifferentiated grade) than cHCC-CC and HCC patients $(69.2 \%$ in ICC vs. $53.3 \%$ in cHCC-CC and $51.5 \%$ in $\mathrm{HCC}, p<0.05)$. No significant difference was found in tumor size among three groups $(P=0.55)$.

\section{Survival analysis and prognosis predictors prior to match}

Until May 2020, a total of 624 (59.9\%) patients died and the median follow-up time was 26.8 months, with $95 \%$ confidence interval (CI) of 22.1 to 31.6 months. Overall, the 1- and 3-year OS rate of cHCCCC, HCC and ICC were $63.9 \%, 86.7 \%, 47.2 \%$ and $48.1 \%, 49.8 \%, 22.6 \%$, respectively, and 5 -year OS rate of cHCC-CC, HCC and ICC was $39.5 \%, 27.8 \%$ and $17.9 \%$, respectively. The median OS time of cHCC-CC, HCC and ICC was 20.5 months, 35.7 months and 11.6 months, respectively $(p<0.0001)$. The survival curve among cHCC-CC, HCC and ICC group prior to match was showed in Figure 2A and B.

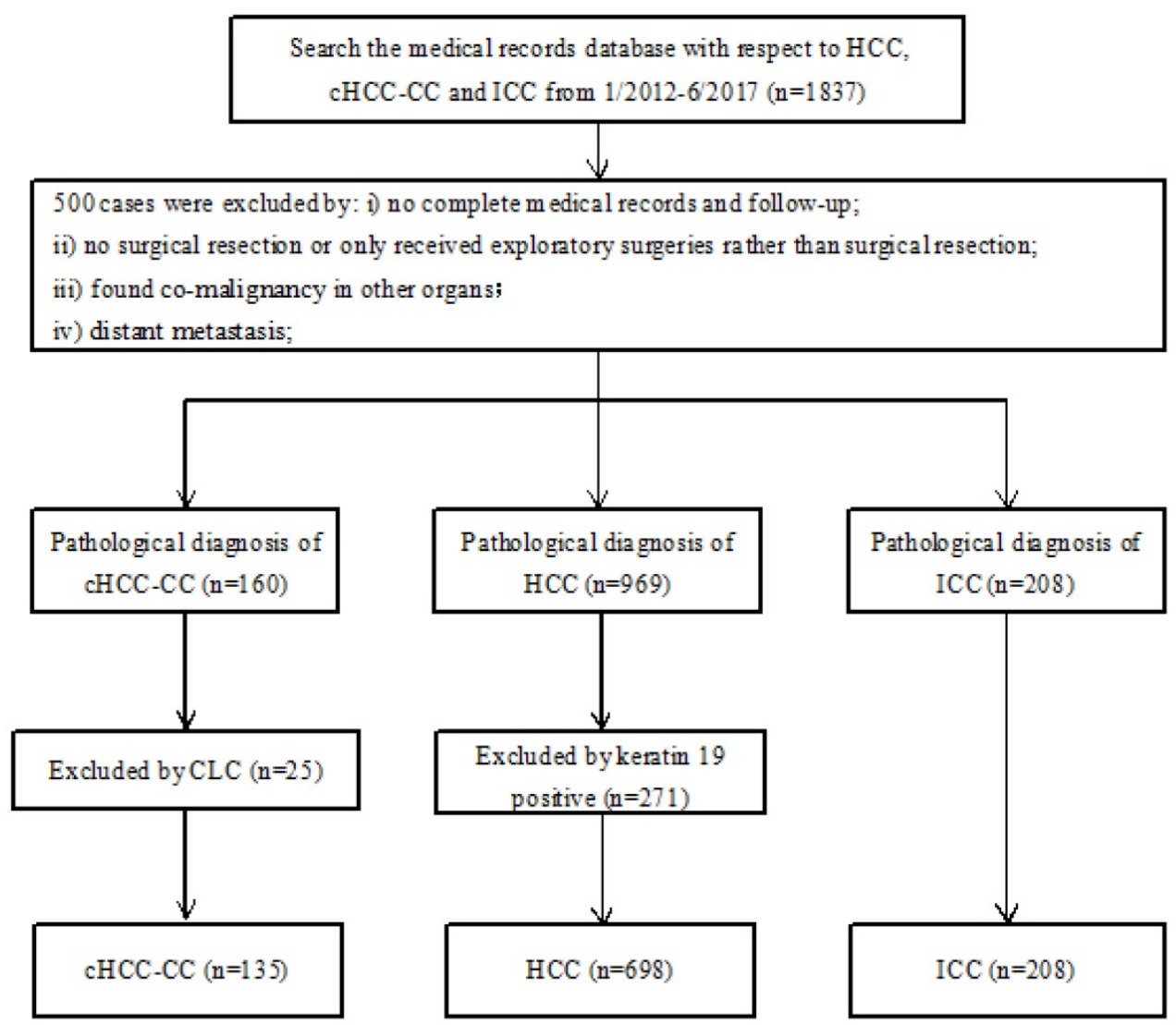

Figure 1. Flow diagram of this study. Abbreviations: HCC: hepatocellular carcinoma; cHCC-CC: combined hepatocellular carcinoma and cholangiocarcinoma; ICC: intrahepatic cholangiocarcinoma; CLC: cholangiolocellular carcinoma. 


\section{Survival curve prior to match}
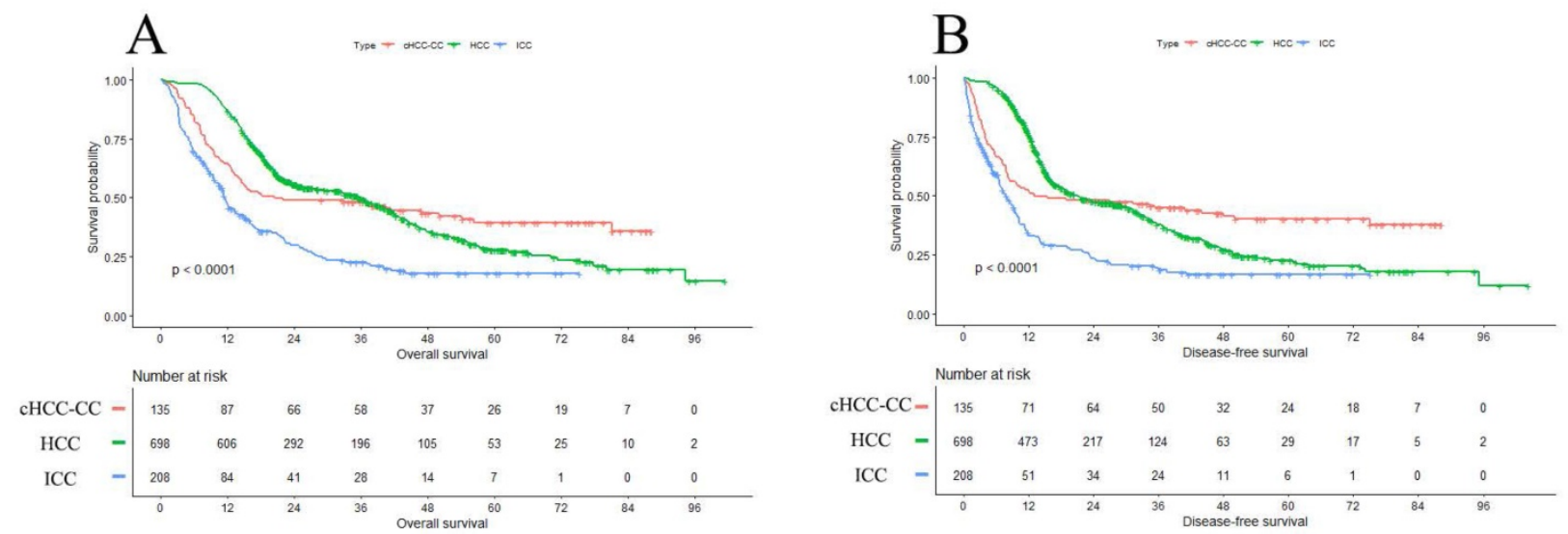

\section{cHCC-CC 1:1 match with HCC}
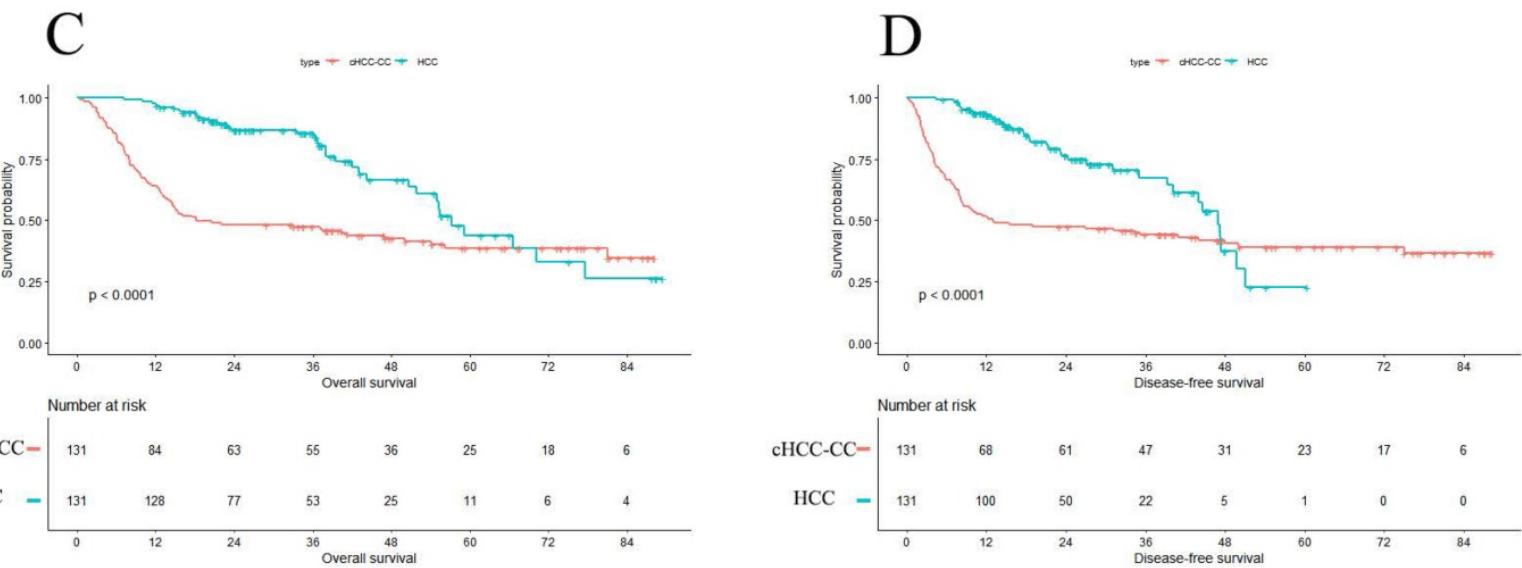

\section{cHCC-CC 1:1 match with ICC}
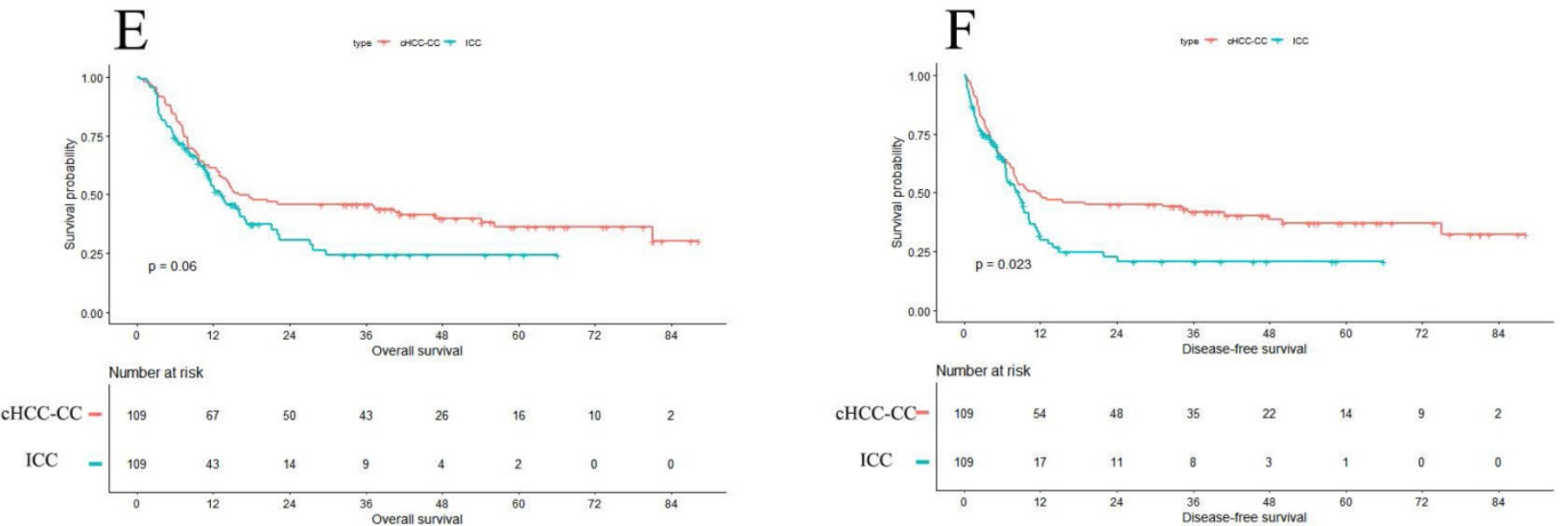

Figure 2. Survival curves prior to match and matched cohorts. A and B: OS and DFS of unmatched cohort. C and D: OS and DFS in cHCC-CC 1:1 matched with HCC cohort. E and F: OS and DFS in CHCC-CC 1:1 matched with ICC cohort. The median overall survival time prior to match of CHCC-CC, HCC and ICC was 20.5 months, 35.7 months and 11.6 months $(p<0.001)$, respectively. The prognosis of $\mathrm{CHCC}-\mathrm{CC}$ were comparable with ICC $(p=0.0599)$, yet worse than $\mathrm{HCC}$ after match $(p<0.001)$. OS: overall survival; DFS: disease-free survival; cHCC-CC: Combined hepatocellular carcinoma and cholangiocarcinoma; HCC: hepatocellular carcinoma; ICC: intrahepatic cholangiocarcinoma.

In multivariate Cox analysis, we found ALB level, tumor size $>5 \mathrm{~cm}$, lymph node infiltration, advanced AJCC stage (III and IV), advanced differentiation grade (poor grade and undifferentiated grade), positive margin, post- operative TACE and tumor type were important risk factors associated with poor prognosis. Besides, we found cHCC-CC had similar prognosis to HCC (HR=1.1, 95\% CI: 0.8-1.4, $p=0.72$ ), but had better prognosis than ICC $(\mathrm{HR}=3.5,95 \%$ CI: 2.6, 4.8, 
$p<0.001)$. The univariate analysis and multivariate Cox analysis prior to match was showed in Table 2 and more details of univariate analysis and multivariate Cox analysis prior to match was displayed in Supplement Table 1.

Table 2. Univariate and multivariate analysis of overall survival prior to match

\begin{tabular}{|c|c|c|c|c|}
\hline \multirow[t]{2}{*}{ Variable } & \multicolumn{2}{|l|}{ Univariate } & \multicolumn{2}{|l|}{ Multivariate } \\
\hline & $\mathrm{HR}(95 \% \mathrm{CI})$ & $p$-value & $\mathrm{HR}(95 \% \mathrm{CI})$ & $p$-value \\
\hline Sex, male & $1.1(0.9,1.3)$ & 0.410 & & \\
\hline $\mathrm{ALB}, \mathrm{g} / \mathrm{L}$ & $1.0(1.0,1.0)$ & 0.005 & $1.0(1.0,1.0)$ & 0.013 \\
\hline \multicolumn{5}{|l|}{ Liver fibrosis } \\
\hline No significant fibrosis & Ref & & Ref & \\
\hline Significant fibrosis & $1.4(1.0,2.0)$ & 0.064 & $1.7(1.2,2.6)$ & 0.008 \\
\hline Advanced fibrosis & $1.7(1.2,2.4)$ & 0.002 & $2.1(1.4,3.1)$ & $<0.001$ \\
\hline Liver cirrhosis & $1.5(1.0,2.2)$ & 0.036 & $1.4(0.9,2.2)$ & 0.124 \\
\hline Tumor size, $>5 \mathrm{~cm}$ & $1.7(1.4,2.0)$ & $<0.001$ & $1.3(1.0,1.6)$ & 0.034 \\
\hline Tumor number, $\geq 2$ & $1.2(1.0,1.4)$ & 0.019 & $1.0(0.7,1.3)$ & 0.825 \\
\hline Satellite lesions, absent & $0.7(0.6,0.8)$ & $<0.001$ & $0.8(0.6,1.1)$ & 0.203 \\
\hline Tumor thrombus, absent & $0.7(0.6,0.8)$ & $<0.001$ & $0.8(0.6,1.0)$ & 0.080 \\
\hline \multicolumn{5}{|l|}{ Lymph node infiltration } \\
\hline Present & Ref & & Ref & \\
\hline Absent & $2.0(1.67,2.5)$ & $<0.001$ & $2.5(1.1,5.0)$ & 0.021 \\
\hline \multicolumn{5}{|l|}{ Differentiation } \\
\hline Well & Ref & & Ref & \\
\hline Moderate & $2.9(1.1,7.7)$ & 0.037 & $3.4(1.1,10.7)$ & 0.039 \\
\hline Poor & $5.6(2.1,14.9)$ & $<0.001$ & $5.8(1.8,18.3)$ & 0.003 \\
\hline Undifferentiated & $19.7(6.7,58.0)$ & $<0.001$ & $22.8(6.4,81.6)$ & $<0.001$ \\
\hline \multicolumn{5}{|l|}{$8^{\text {th }}$ AJCC stage } \\
\hline I & Ref & & Ref & \\
\hline II & $0.8(0.6,1.1)$ & 0.172 & $0.6(0.4,0.9)$ & 0.022 \\
\hline III & $1.8(1.5,2.1)$ & $<0.001$ & $1.4(1.0,2.1)$ & 0.044 \\
\hline IV & $2.8(2.1,3.8)$ & $<0.001$ & $2.6(1.9,3.7)$ & $<0.001$ \\
\hline Transfusion, yes & $0.6(0.5,0.8)$ & $<0.001$ & $0.9(0.7,1.2)$ & 0.343 \\
\hline Blood loss, $>400 \mathrm{ml}$ & $1.4(1.2,1.6)$ & $<0.001$ & $1.2(1.0,1.5)$ & 0.059 \\
\hline Margin, R1 & $1.7(1.4,2.2)$ & $<0.001$ & $1.7(1.3,2.1)$ & $<0.001$ \\
\hline Anatomy resection & $0.8(0.7,0.9)$ & 0.002 & $0.9(0.7,1.1)$ & 0.277 \\
\hline Postoperative TACE & $1.8(1.6,2.2)$ & $<0.001$ & $1.8(1.5,2.2)$ & $<0.001$ \\
\hline \multicolumn{5}{|l|}{ Tumor type } \\
\hline cHCC-CC & Ref & & Ref & \\
\hline $\mathrm{HCC}$ & $1.0(0.8,1.2)$ & 0.799 & $0.9(0.6,1.2)$ & 0.397 \\
\hline ICC & $2.3(1.8,3.1)$ & $<0.001$ & $3.5(2.5,4.9)$ & $<0.001$ \\
\hline
\end{tabular}

Abbreviation: cHCC-CC: Combined hepatocellular carcinoma and cholangiocarcinoma; HCC: hepatocellular carcinoma; ICC: intrahepatic

cholangiocarcinoma; HR: hazard ratio; CI: confidence interval; Ref: reference; ALB: albumin; AJCC: American Joint Committee on Cancer; TACE: transhepatic arterial chemotherapy and embolization; HR: hazard ratio; CI: confidence interval; ref: reference.

\section{Survival analysis and prognosis predictors after matching on ALB level, tumor size, lymph node infiltration, AJCC stage and margin}

Since most of the risk factors found in unmatched cohort except tumor size were significantly different among cHCC-CC, HCC and ICC group, we conducted a further analysis by two separated matched cohorts (cHCC-CC 1:1 matched with HCC; and cHCC-CC 1:1 matched with ICC) using propensity score matching on ALB level, tumor size, lymph node infiltration, AJCC stage, differentiation, margin and postoperative TACE.
After matching, most of the above variables were found no significant difference in two matched cohorts, except for differentiation (cHCC-CC versus HCC, $p=0.0028$; cHCC-CC versus ICC, $p=0.016$ ), margin (cHCC-CC versus HCC, $p=0.046$ ) and postoperative TACE (cHCC-CC versus HCC, $p<0.0001$ ) (Table 3).

After matching, survival curves regarding to OS and DFS of two matched cohorts were presented in Figure 2. The OS of $\mathrm{CHCC}-\mathrm{CC}$ were significantly worse than HCC $(p<0.001)$ but comparable to ICC $(p=0.0599)$. The median OS time of two cohorts were (cHCC-CC versus HCC, 18.2 months versus 57.3 months, $p<0.001$; cHCC-CC versus ICC, 16.1 months versus 13.1 months, $p=0.06)$. The DFS of cHCC-CC were significantly worse than HCC $(p<0.001)$, however better than ICC $(p=0.0229)$. The median DFS time of two cohorts were (cHCC-CC vs. HCC, 13.0 months versus 46.9 months, $p<0.001$; $\mathrm{cHCC}-\mathrm{CC}$ vs. ICC, 11.3 months versus 8.6 months, $p=0.0229$ ).

In multivariate Cox analysis of matched cohorts, we found positive lymph node infiltration (HR: 2.5, 95\%CI: 1.1-5.0, $p=0.025)$ and no postoperative TACE (HR: 2.8, 95\%CI: 1.8- 4.3, $p<0.001$ ) were relating to poor prognosis in both cHCC-CC 1:1 matched HCC cohort and cHCC-CC 1:1 matched ICC cohort. The univariate analysis and multivariate Cox regression analysis of two matched cohorts were showed in Table 4.

While stratified by negative lymph node infiltration of two matched cohorts, cHCC-CC patients had significantly poor overall survival and disease-free survival than HCC patients (OS: cHCC-CC vs. HCC, $p<0.001$ ) but similar to ICC (OS and DFS, cHCC-CC vs. ICC, $p=0.41$ and $p=0.21$, respectively) (Figure 3a). However, when stratified by positive lymph node infiltration of two matched cohorts, the OS rate and DFS rate of cHCC-CC were similar to HCC but better than ICC (Figure 3b). The median overall survival time of CHCC-CC stratified by lymph node (LN) infiltration were significantly different (median overall survival time, LN positive vs. LN negative: 22.1 months vs. 12.9 months, $p=0.019$ ) (Supplement Figure 1A).

In no postoperative TACE patients of two matched cohorts, the prognosis of CHCC-CC were better than ICC (median overall survival time, cHCCCC vs. ICC: 15.0 months vs. 9.6 months, $p=0.0002$ ) but worse than HCC (median overall survival time, cHCC-CC vs. HCC: 15.2 months vs. 55.4 months, $p=0.0025$ ) (Figure 3c). In patients receiving postoperative TACE of two matched cohorts, cHCC-CC had significantly poor overall survival than HCC $(p=0.028)$ but similar to ICC $(p=0.2)$ (Figure 3d). The mean overall survival time of CHCC-CC stratified 
by postoperative TACE were significantly different (mean overall survival time, TACE vs. no TACE: 52.0 months vs. 35.2 months, $p=0.012$ ) (Supplement Figure 1C).

Table 3. Comparison among Patients with $\mathrm{CHCC}-\mathrm{CC}, \mathrm{HCC}$ and ICC when matched on ALB, tumor size, lymph node infiltration, AJCC stage and margin

\begin{tabular}{|c|c|c|c|c|c|c|}
\hline \multirow[t]{2}{*}{ Variable } & \multicolumn{3}{|l|}{ 1:1 match } & \multicolumn{3}{|l|}{ 1:1 match } \\
\hline & cHCC-CC $(n=131)$ & $\mathrm{HCC}(\mathrm{n}=131)$ & $p$-value & cHCC-CC $(n=109)$ & ICC $(n=109)$ & $p$-value \\
\hline Sex, male, n (\%) & $114(87)$ & $111(84.7)$ & 0.72 & $95(87.2)$ & 69 (63.3) & $<0.01$ \\
\hline Age, year, $\leq 60$ & $102(77.9)$ & $110(84)$ & 0.27 & $83(76.1)$ & $71(65.1)$ & 0.10 \\
\hline Hypertension, n (\%) & $13(9.9)$ & $12(9.2)$ & 1.00 & $10(9.2)$ & $16(14.7)$ & 0.30 \\
\hline Diabetes mellitus, n (\%) & $9(6.9)$ & $9(6.9)$ & 1.00 & $7(6.4)$ & $4(3.7)$ & 0.54 \\
\hline Hepatitis, n (\%) & $66(50.4)$ & $95(72.5)$ & 0.0004 & $55(50.5)$ & $49(45)$ & 0.50 \\
\hline Hypersplenia, n (\%) & $14(10.7)$ & $14(10.7)$ & 1.00 & $12(11)$ & $7(6.4)$ & 0.34 \\
\hline $\mathrm{ALT}, \mathrm{U} / \mathrm{L}$, mean $\pm \mathrm{SD}$ & $54.37 \pm 95.48$ & $58.22 \pm 60.64$ & 0.70 & $47.25 \pm 34.64$ & $47.57 \pm 63.21$ & 0.96 \\
\hline $\mathrm{AST}, \mathrm{U} / \mathrm{L}$, mean $\pm \mathrm{SD}$ & $61.13 \pm 131.24$ & $62.10 \pm 58.04$ & 0.94 & $49.97 \pm 38.71$ & $50.13 \pm 60.81$ & 0.98 \\
\hline $\mathrm{ALB}, \mathrm{g} / \mathrm{L}$, mean $\pm \mathrm{SD}$ & $41.9 \pm 4.6$ & $41.9 \pm 4.2$ & 0.98 & $41.7 \pm 4.4$ & $39.8 \pm 8.4$ & 0.93 \\
\hline $\mathrm{TB}, \mathrm{umol} / \mathrm{L}$, mean $\pm \mathrm{SD}$ & $15.86 \pm 9.84$ & $15.49 \pm 10.07$ & 0.77 & $15.97 \pm 9.71$ & $18.54 \pm 22.93$ & 0.28 \\
\hline $\mathrm{PT}, \mathrm{s}$, mean $\pm \mathrm{SD}$ & $12.01 \pm 1.91$ & $12.34 \pm 1.40$ & 0.18 & $11.99 \pm 1.92$ & $12.07 \pm 1.62$ & 0.79 \\
\hline $\mathrm{INR}$, mean $\pm \mathrm{SD}$ & $1.04 \pm 0.16$ & $1.07 \pm 0.12$ & 0.15 & $1.04 \pm 0.16$ & $1.04 \pm 0.14$ & 0.82 \\
\hline $\mathrm{AFP}, \mathrm{ng} / \mathrm{ml}$, mean $\pm \mathrm{SD}$ & $231.6 \pm 406.0$ & $440.5 \pm 433.5$ & 0.002 & $106.08 \pm 245.92$ & $311.55 \pm 406.11$ & $<0.0001$ \\
\hline Ca19-9, U/ml, mean \pm SD & $92.81 \pm 226.09$ & $23.99 \pm 26.21$ & 0.0021 & $100.11 \pm 579.37$ & $94.91 \pm 252.25$ & 0.95 \\
\hline $\mathrm{Ca} 125, \mathrm{U} / \mathrm{ml}$, mean $\pm \mathrm{SD}$ & $87.10 \pm 531.45$ & $74.81 \pm 465.68$ & 0.87 & $6.53 \pm 31.29$ & $24.78 \pm 114.66$ & 0.12 \\
\hline $\mathrm{CEA}, \mathrm{ng} / \mathrm{ml}$, mean $\pm \mathrm{SD}$ & $5.92 \pm 28.51$ & $4.21 \pm 17.38$ & 0.59 & $47.25 \pm 34.64$ & $47.57 \pm 63.21$ & 0.9 \\
\hline Liver fibrosis, n (\%) & & & $<0.0001$ & & & 0.0045 \\
\hline No significant fibrosis & $19(14.5)$ & $3(2.5)$ & & $16(14.7)$ & $18(16.5)$ & \\
\hline Significant fibrosis & $19(14.5)$ & $42(35.3)$ & & $15(13.8)$ & $20(18.3)$ & \\
\hline Advanced fibrosis & $41(31.3)$ & $64(53.8)$ & & $33(30.3)$ & $50(45.9)$ & \\
\hline liver cirrhosis & $52(39.7)$ & $10(8.4)$ & & 45 (41.3) & $21(19.3)$ & \\
\hline Tumor size, $>5 \mathrm{~cm}, \mathrm{n}(\%)$ & $87(66.4)$ & $101(77.1)$ & 0.074 & 69 (63.3) & $74(71.2)$ & 0.28 \\
\hline Tumor number, single, $\mathrm{n}(\%)$ & $59(45)$ & $68(51.9)$ & 0.32 & $52(47.7)$ & $65(59.6)$ & 0.10 \\
\hline Satellite lesions, n (\%) & $44(33.6)$ & $44(33.6)$ & 1.00 & $34(31.2)$ & $27(24.8)$ & 0.37 \\
\hline Tumor capsule, $\mathrm{n}(\%)$ & $23(17.6)$ & $29(22.1)$ & 0.37 & $18(16.5)$ & $35(32.1)$ & $<0.0001$ \\
\hline Tumor thrombus, n (\%) & $47(35.9)$ & $92(70.2)$ & $<0.0001$ & $34(31.2)$ & $30(27.5)$ & 0.66 \\
\hline Lymph node infiltration, $\mathrm{n}(\%)$ & $14(10.7)$ & $14(10.7)$ & 1.00 & $18(16.5)$ & $18(16.5)$ & 1.00 \\
\hline Differentiation, $\mathrm{n}(\%)$ & & & 0.0028 & & & 0.016 \\
\hline Well & $5(3.8)$ & $1(0.8)$ & & $4(3.7)$ & $4(3.7)$ & \\
\hline Moderate & $55(42)$ & $57(45.2)$ & & $48(44)$ & $26(23.9)$ & \\
\hline Poor & $60(45.8)$ & $68(54)$ & & $54(49.5)$ & $73(67)$ & \\
\hline Undifferentiated & $11(8.4)$ & $0(0)$ & & $3(2.8)$ & $6(5.5)$ & \\
\hline $8^{\text {th }}$ AJCC stage, n (\%) & & & 1.00 & & & 1.00 \\
\hline I & $11(8.4)$ & $11(8.4)$ & & $11(10.1)$ & $11(10.1)$ & \\
\hline II & $32(24.4)$ & $32(24.4)$ & & $32(29.4)$ & $32(29.4)$ & \\
\hline III & $74(56.5)$ & $74(56.5)$ & & $48(44)$ & $48(44)$ & \\
\hline IV & $14(10.7)$ & $14(10.7)$ & & $18(16.5)$ & $18(16.5)$ & \\
\hline T stage, $\mathbf{n}(\%)$ & & & 0.013 & & & 0.76 \\
\hline $\mathrm{T} 1$ & 15 (11.5) & 14 (10.7) & & 15 (13.8) & 16 (14.7) & \\
\hline $\mathrm{T} 2$ & $35(26.7)$ & $32(24.4)$ & & $35(32.1)$ & $32(29.4)$ & \\
\hline T3 & 48 (36.6) & $29(22.1)$ & & 39 (35.8) & 35 (32.1) & \\
\hline $\mathrm{T} 4$ & $33(25.2)$ & $56(42.7)$ & & $20(18.3)$ & $26(23.9)$ & \\
\hline N stage, n (\%) & & & 1.00 & & & 1.00 \\
\hline No & $117(89.3)$ & $117(89.3)$ & & $91(83.5)$ & $91(83.5)$ & \\
\hline N1 & $14(10.7)$ & $14(10.7)$ & & $18(16.5)$ & $18(16.5)$ & \\
\hline Transfusion, $\mathrm{n}(\%)$ & $18(13.7)$ & $11(8.7)$ & 0.27 & $16(14.7)$ & $9(8.5)$ & 0.23 \\
\hline Blood loss, $\leq 400 \mathrm{ml}, \mathrm{n}(\%)$ & $77(58.8)$ & $90(70.9)$ & 0.057 & $66(60.6)$ & $84(79.2)$ & 0.0046 \\
\hline Margin, R0, n (\%) & $108(82.4)$ & $119(91.5)$ & 0.046 & $87(79.8)$ & $62(70.5)$ & 0.18 \\
\hline Surgical method, $\mathrm{n}(\%)$ & & & 0.0772 & & & 0.0003 \\
\hline Major resection & $59(45)$ & $73(55.7)$ & & $53(48.6)$ & $68(62.4)$ & \\
\hline Minor resection & $63(48.1)$ & $44(33.6)$ & & $50(45.9)$ & $25(22.9)$ & \\
\hline Resection+ Ablation & $6(4.6)$ & $11(8.4)$ & & $4(3.7)$ & $15(13.8)$ & \\
\hline Liver transplantation & $3(2.3)$ & $3(2.3)$ & & $2(1.8)$ & $1(0.9)$ & \\
\hline Anatomy resection, n (\%) & $54(42.9)$ & $66(52.4)$ & 0.17 & $49(46.7)$ & $72(72.7)$ & 0.0003 \\
\hline Postoperative TACE, n (\%) & $39(29.8)$ & $83(63.4)$ & $<0.0001$ & $31(28.4)$ & $39(35.8)$ & 0.31 \\
\hline Disease-free survival, $\mathrm{m}$, mean $\pm \mathrm{SD}$ & $28.5 \pm 28.1$ & $22.4 \pm 13.1$ & 0.025 & $25.7 \pm 25.8$ & $9.3 \pm 12.6$ & $<0.0001$ \\
\hline Overall survival, $\mathrm{m}$, mean $\pm \mathrm{SD}$ & $31.9 \pm 27.2$ & $33.3 \pm 18.1$ & 0.62 & $29.4 \pm 25.3$ & $13.7 \pm 12.9$ & $<0.0001$ \\
\hline
\end{tabular}


Table 4. Univariate and multivariate analysis of overall survival of two matched cohorts

\begin{tabular}{|c|c|c|c|c|c|c|}
\hline \multirow[t]{2}{*}{ Variable } & \multicolumn{3}{|c|}{ 1:1 match of $\mathrm{cHCC}-\mathrm{CC}(\mathrm{n}=131)$ and HCC $(\mathrm{n}=131)$} & \multicolumn{3}{|c|}{ 1:1 match of cHCC-CC $(n=109)$ and ICC $(n=109)$} \\
\hline & Univariate: $p$ value & Multivariate: HR (95\% CI) & $p$-value & Univariate: $p$ value & Multivariate: HR (95\% CI) & $p$-value \\
\hline Sex, male & 0.197 & & & 0.994 & & \\
\hline $\mathrm{ALB}, \mathrm{g} / \mathrm{L}$ & 0.991 & & & 0.554 & & \\
\hline \multicolumn{7}{|l|}{ Tumor size } \\
\hline$\leq 5 \mathrm{~cm}$ & Ref & & & Ref & Ref & Ref \\
\hline$>5 \mathrm{~cm}$ & 0.949 & & & $<0.001$ & $1.3(0.8,2.3)$ & 0.288 \\
\hline Satellite lesions & 0.216 & & & $<0.001$ & $0.8(0.4,1.4)$ & 0.405 \\
\hline Tumor thrombus & 0.816 & & & $<0.001$ & $0.7(0.4,1.2)$ & 0.161 \\
\hline \multicolumn{7}{|l|}{$\begin{array}{l}\text { Lymph node } \\
\text { infiltration }\end{array}$} \\
\hline Negative & Ref & Ref & Ref & Ref & Ref & Ref \\
\hline Positive & $<0.001$ & $2.5(1.1,5.0)$ & 0.025 & 0.029 & $1.7(1.1,2.5)$ & 0.028 \\
\hline \multicolumn{7}{|l|}{ Differentiation } \\
\hline Well & Ref & Ref & Ref & Ref & Ref & Ref \\
\hline Moderate & 0.594 & $2.5(0.3,18.8)$ & 0.378 & 0.305 & $2.2(0.3,16.9)$ & 0.459 \\
\hline Poor & 0.090 & $8.2(1.1,61.3)$ & 0.040 & 0.012 & $3.8(0.5,29.8)$ & 0.203 \\
\hline \multicolumn{7}{|l|}{$8^{\text {th }}$ AJCC stage } \\
\hline I & Ref & Ref & Ref & Ref & Ref & Ref \\
\hline II & 0.097 & $0.5(0.2,1.1)$ & 0.084 & 0.933 & $1.7(0.5,5.7)$ & 0.367 \\
\hline III & 0.801 & $1.3(0.6,2.8)$ & 0.480 & 0.015 & $2.3(0.7,7.3)$ & 0.164 \\
\hline IV & 0.018 & $2.6(1.1,6.0)$ & 0.025 & 0.014 & $2.9(0.9,9.7)$ & 0.082 \\
\hline Margin, R1 & 0.020 & $2.3(1.3,4.1)$ & 0.005 & 0.755 & $0.8(0.4,1.5)$ & 0.466 \\
\hline \multicolumn{7}{|l|}{ Surgical method } \\
\hline Major resection & Ref & Ref & Ref & Ref & Ref & Ref \\
\hline Minor resection & 0.061 & $0.6(0.4,0.9)$ & 0.015 & 0.002 & $0.9(0.5,1.8)$ & 0.829 \\
\hline Resection+ Ablation & 0.033 & $2.2(0.6,7.7)$ & 0.237 & 0.109 & $0.8(0.2,3.5)$ & 0.720 \\
\hline Liver transplantation & 0.532 & $0.3(0.1,1.4)$ & 0.127 & 0.322 & $0.4(0.1,2.7)$ & 0.997 \\
\hline \multicolumn{7}{|c|}{ Postoperative TACE } \\
\hline Yes & Ref & Ref & Ref & Ref & Ref & Ref \\
\hline No & $<0.001$ & $2.8(1.8,4.3)$ & $<0.001$ & $<0.001$ & $2.5(1.4,4.5)$ & 0.003 \\
\hline \multicolumn{7}{|l|}{ Tumor type } \\
\hline cHCC-CC & Ref & Ref & Ref & Ref & Ref & Ref \\
\hline $\mathrm{HCC}$ & $<0.001$ & $0.3(0.2,0.6)$ & $<0.001$ & - & - & - \\
\hline ICC & - & - & - & 0.061 & $1.3(0.9,2.0)$ & 0.129 \\
\hline
\end{tabular}

Abbreviation: cHCC-CC: Combined hepatocellular carcinoma and cholangiocarcinoma; HCC: hepatocellular carcinoma; ICC: intrahepatic cholangiocarcinoma; HR: hazard ratio; CI: confidence interval; Ref: reference; ALB: albumin; AJCC: American Joint Committee on Cancer; TACE: transhepatic arterial chemotherapy and embolization; HR: hazard ratio; CI: confidence interval; ref: reference.

\section{Discussion}

cHCC-CC is a distinct entity of primary liver tumor, with a proportion of about $0.8-6.5 \%$ in primary liver malignancy $[2,6,15,17,18]$. However, the results of previous studies regarding to outcomes of cHCC-CC were inconsistent. In the present study, we found the long-term prognosis of cHCC-CC was worse than HCC, however, better than ICC in the whole cohort, but the long term overall survival of cHCC-CC was worse than HCC, yet similar to ICC in matched cohorts. Besides, the multivariate cox regression analysis within matched cohorts revealed that the independent prognosis risk factors of cHCCCC were lymph node infiltration and postoperative TACE.

The clinical characteristics and prognosis of cHCC-CC compared to HCC and ICC were inconsistent, albeit it had been discussed for decades. In this study, we found baseline clinical characteristics of cHCC-CC were comparable with HCC regarding to age, gender, and incidence of hypertension, diabetes mellitus and cirrhosis. These findings were in consistent with previous studies which found a similar incidence of demographic characteristics and cirrhosis between cHCC-CC and HCC $[4,11,12,23]$. Besides, the incidence of hepatitis B or C of cHCC-CC was similar to ICC $(p=0.197)$ but different from HCC $(p<0.001)$. This was in consistent with Jarnagin et al. which found that the rate of hepatitis B or $C$ between cHCC-CC and ICC was similar, with an incidence of $15 \%$ in cHCC-CC patients [5]. cHCC-CC showed an intermediate prognosis between HCC and ICC prior to match (median overall survival time: cHCC-CC, HCC and ICC, 20.5 months, 35.7 months and 11.6 months, $p<0.001$ ), and a comparable prognosis with ICC, yet worse than HCC in matched cohorts (median overall survival time: $\mathrm{CHCC}-\mathrm{CC}$ versus ICC, 16.1 months versus 13.1 months, $p=0.06$; $\mathrm{cHCC}-\mathrm{CC}$ versus HCC, 18.2 months versus 57.3 months, $p<0.001$ ). This can be demonstrated by previous study which reported the prognosis of CHCC-CC was worse than HCC, however, comparable with ICC, with median overall survival time of 6.0 months, 17.4 months, and 
4.4 months in cHCC-CC, HCC and ICC, respectively [12]. Another population-based study suggested that the 5-year OS rate of CHCC-CC, HCC and ICC was $34.4 \%, 43.5 \%$ and $33.3 \%$, respectively [15]. The clinical characteristics and prognosis of CHCC-CC were different from HCC and ICC, suggesting that $\mathrm{CHCC}$ $\mathrm{CC}$ was a distinct entity of primary liver malignancy and should be treated individually.

Lymph node infiltration was associated with poor survival of cHCC-CC after surgery [15, 24]. In the present study, the incidence of lymph node infiltration in cHCC-CC was $13.3 \%$ and patients with positive LN infiltration survived significant poor prognosis than those negative (median overall



C

No postoperative TACE

cHCC-CC 1:1 match with HCC

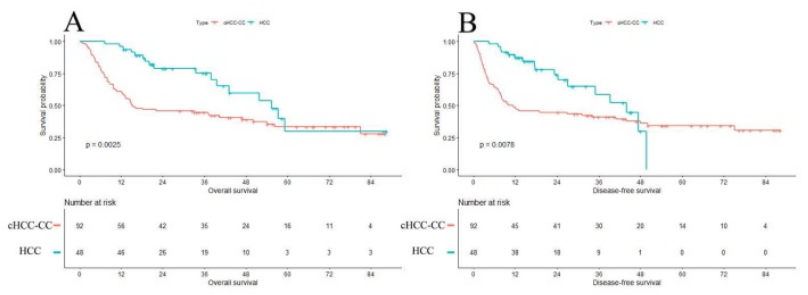

cHCC-CC 1:1 match with ICC
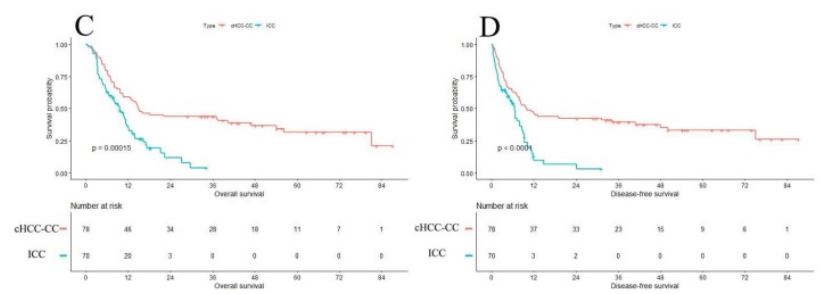

survival time, positive vs. negative, 22.1 months vs. 12.9 months, $p=0.019)$. Even in 1:1 matched cohort with HCC, cHCC-CC still had poor prognosis than HCC either in LN negative (median overall survival time, cHCC-CC vs. HCC, 22.1 months vs. 66.6 months, $p<0.001$ ) or $\mathrm{LN}$ positive patients (median overall survival time, cHCC-CC vs. HCC, 11.1 months vs. 18.7 months, $p=0.937$ ) (Figure 3a, 3b). Previous study found the incidence of lymph node metastasis in cHCC-CC was about $8.3 \%-60.0 \%[6,13,14,25]$, with an average rate of $48 \%$ [26]. In further, cHCC-CC patients with positive LN infiltration suffered an unfavorable average overall survival time of 7.8 months than those of 20.2 months in negative LN [13].
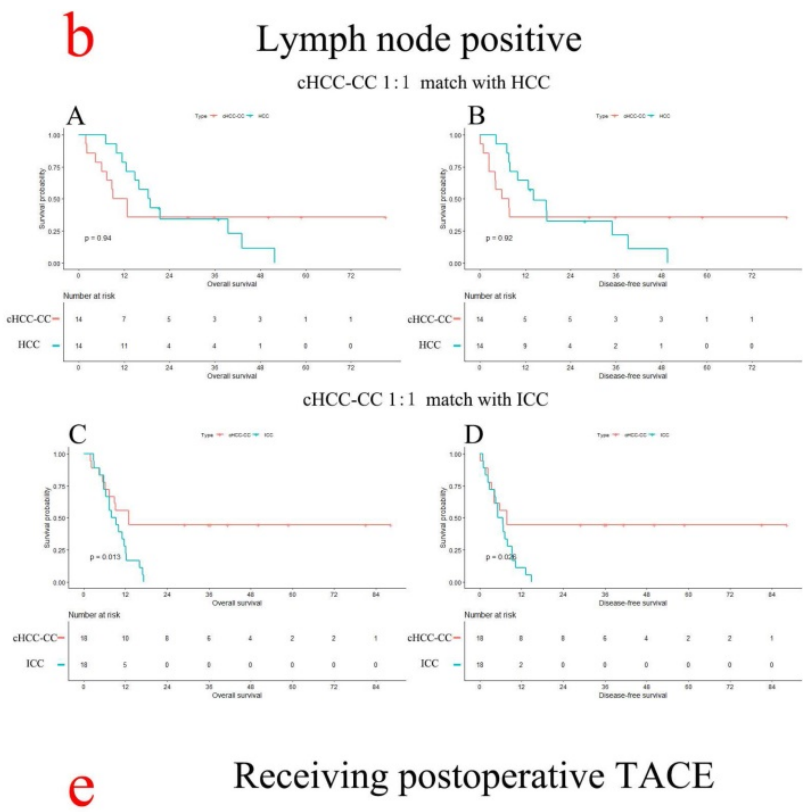

cHCC-CC 1:1 match with HCC

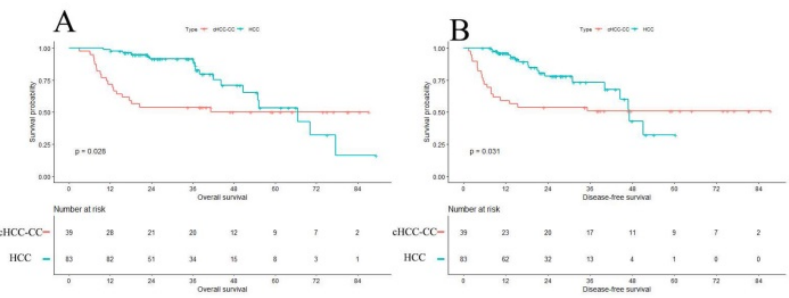

cHCC-CC 1:1 match with ICC

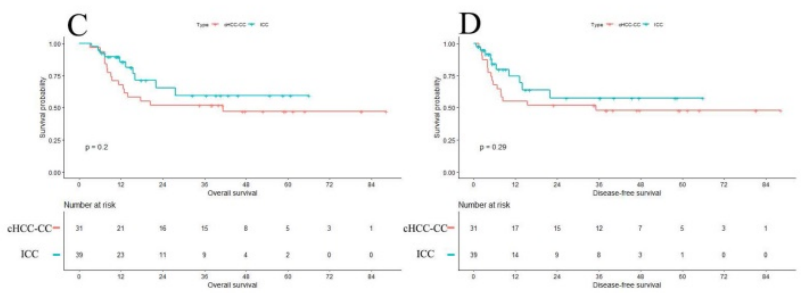

Figure 3. Survival curves of matched cohorts stratified by LN infiltration and postoperative TACE. A-D: The OS and DFS in cHCC-CC 1:1 matched with HCC cohort and cHCC-CC 1:1 matched with ICC cohort, respectively. a and b: Survival curves of matched cohorts stratified by LN infiltration; c and d: Survival curves of matched cohorts stratified by postoperative TACE. cHCC-CC had significantly worse prognosis than HCC in LN negative patient $(p<0.001)$ but better prognosis than ICC in LN positive patient $(p<0.05)$. cHCC had significant worse prognosis than $\mathrm{HCC}$ in either receiving or not receiving postoperative TACE patients $(p<0.05)$, but have better prognosis than ICC in no postoperative TACE patients $(p<0.001)$. LN: lymph node; TACE: transhepatic arterial chemotherapy and embolization; OS: overall survival; DFS: disease-free survival; cHCC-CC: Combined hepatocellular carcinoma and cholangiocarcinoma; HCC: hepatocellular carcinoma; ICC: intrahepatic cholangiocarcinoma. 
Another population-based study suggested that lymph node status of cHCC-CC were strongly associated with overall survival, with a remarkable increased risk of death in positive LN patients [15]. A recent systematic review of cHCC-CC revealed that LN metastasis was strongly associated with decreased overall survival after surgical resection, with a hazards ratio of 2.84, $p<0.0001$ [26]. As LN metastasis resulted in worse prognosis than negative LN and lymphadenectomy was only performed in about one third of cHCC-CC patient [15], lymphadenectomy should be regarded as a routine procedure for suspected cHCC-CC following curative surgery.

Postoperative TACE may benefit for OS and DFS for cHCC-CC after surgical resection. In this study, although cHCC-CC patients obtain significant worse prognosis than HCC patients in either receiving or not receiving postoperative TACE $(p<0.05)$, cHCC-CC patients who received postoperative TACE still obtained a favorable prognosis than those who didn't (mean overall survival time, TACE vs. no TACE: 52.0 months vs. 35.2 months, $p=0.012$ ). TACE was considered as an alternative treatment method of advanced or unresectable HCC or an adjuvant therapy following surgical resection [27-29]. Previous studies reported that TACE served as an adjuvant method which prolonged survival of HCC following surgical resection, with a 5-year OS rate of $53.3 \%$ in patients receiving more than twice TACE treatments [30]. Moreover, Kim et al. reported that TACE could significantly improve the OS of unresectable cHCCCC, with a favorable median OS time of 12.3 months [31]. As we know, tumor hypovascularity was the major factor restricting the response of TACE [24, 31]. Although some studies insisted that CHCC-CC was hypovascular and TACE was not recommended [32, 33], the rate of hypervascularity in cHCC-CC in previous study reached about $40.9 \%-80 \%$ [31, 34], which enabled a favorable feasibility and effect of applying postoperative TACE for cHCC-CC. Thus, more studies regarding to the proportion of tumor arterialization could be conducted to verify the effect of TACE in CHCC-CC.

Sorafenib therapy, as an alternative treatment method, was used in many advanced primary liver malignancies. A recent systematic review showed that sorafenib was not superior to hepatic arterial infusion chemotherapy in advanced HCC patients, because that sorafenib was associated with diarrhea and hand-foot syndrome [35]. In addition, capecitabine was recommended as an alternative adjuvant chemotherapy following surgical resection of biliary tract cancer [36]. As for cHCC-CC, only one study that reported the use of molecular targeted therapy, but failed to demonstrate the superiority of molecular targeted therapy in terms of recurrence or survival [37]. In the present study, we found that only few patient $(<5 \%)$ received sorafenib treatment after surgical resection and whether the others were being treated by sorafenib was unknown. So we didn't include this variable in the study. This was also a limitation resulting from the nature of retrospective study.

The study has several strengths. First, this study enrolled a large cohort of patients with long-term follow-up and histological evidence. Second, the definition of cHCC-CC in most of the previous study was vague; however, in the present study, we adopted the latest classification (2019 WHO) and removed patients who were diagnosed as cholangiolocellular carcinoma (CLC). Third, we applied propensity score matching analysis, to reduce the bias of potential confounding in baseline characteristics and the groups were well matched. Forth, although the benefit of TACE for cHCC-CC remained debatable in many previous studies,[31-33] this study found that postoperative TACE could benefit for survival for cHCC-CC after surgical resection.

Two limitations should be mentioned in this study. First, the nature of retrospective study may generate selective bias. Second, although the intention of propensity score matching of baseline variables was to reduce differences between groups, a decreased sample size will appear when increasing matched variables. Thus, in this study, we only matched variables relating to prognosis and most of cHCC-CC patients were enrolled in two matched cohorts, and only few cHCC-CC patients were excluded in matched cohorts.

\section{Conclusion}

The baseline clinical features and prognosis of CHCC-CC were inconsistent with HCC and ICC, and should be considered as a distinct entity of primary liver malignancy. The long term survival of cHCC-CC was worse than HCC, yet comparable with ICC when matched on ALB level, tumor size, lymph node infiltration, AJCC stage and margin. Presence of LN infiltration and no postoperative TACE were associated with poor prognosis of $\mathrm{CHCC}-\mathrm{CC}$.

\section{Abbreviations}

cHCC-CC: Combined hepatocellular carcinoma and cholangiocarcinoma; HCC: hepatocellular carcinoma; ICC: intrahepatic cholangiocarcinoma; WHO: World Health Organization; CLC: cholangiolocellular carcinoma; OS: overall survival; DFS: diseasefree survival; HR: hazard ratio; CI: confidence interval; Ref: reference; ALT: alanine amino- 
transferase; SD: standard deviation; AST: aspartate aminotransferase; ALB: albumin; TB: total bilirubin; PT: prothrombin time; INR: International Normalized Ratio; AFP: alpha fetoprotein; CEA: carcinoembryonic antigen; AJCC: American Joint Committee on Cancer; LN: lymph node; TACE: transhepatic arterial chemotherapy and embolization.

\section{Supplementary Material}

Supplementary figures and tables.

http://www.medsci.org/v18p0187s1.pdf

\section{Acknowledgments}

\section{Author contributions}

Study concept and design: TYY, WLY; Acquisition of data: TYY, TF; Analysis and interpretation: ZT and ZYN; Draft the manuscript and preliminary revise: TYY; Study supervision and final approval: CZY.

\section{Availability of data and material}

The clinical data in this study is available from the corresponding author on reasonable request.

\section{Ethics approval and inform consent}

This study was approved by the Ethics Committee of West China Hospital of Sichuan University. And inform consent was waived because no individual information was disclosed in this study.

\section{Competing Interests}

The authors have declared that no competing interest exists.

\section{References}

1. Chang CC, Chen YJ, Huang T, Chen C, Kuo F, Eng H, et al. Living Donor Liver Transplantation for Combined Hepatocellular Carcinoma and Cholangiocarcinoma: Experience of a Single Center. Annals of transplantation. 2017; 22: 115-20.

2. Garancini M, Goffredo P, Pagni F, Romano F, Roman S, Sosa JA, et al. Combined hepatocellular-cholangiocarcinoma: a population-level analysis of an uncommon primary liver tumor. Liver transplantation: official publication of the American Association for the Study of Liver Diseases and the International Liver Transplantation Society. 2014; 20: 952-9.

3. Chu KJ, Lu C, Dong H, Fu X, Zhang H, Yao X. Hepatitis B virus-related combined hepatocellular-cholangiocarcinoma: clinicopathological and prognostic analysis of 390 cases. European journal of gastroenterology \& hepatology. 2014; 26: 192-9.

4. Yin X, Zhang B-H, Qiu S-J, Ren Z-G, Zhou J, Chen X-H, et al. Combined Hepatocellular Carcinoma and Cholangiocarcinoma: Clinical Features, Treatment Modalities, and Prognosis. Annals of Surgical Oncology. 2012; 19: 2869-76.

5. Jarnagin WR, Weber S, Tickoo SK, Koea JB, Obiekwe S, Fong $\mathrm{Y}$, et al. Combined hepatocellular and cholangiocarcinoma. Cancer. 2002; 94: 2040-6.

6. Koh KC, Lee H, Choi MS, Lee JH, Paik SW, Yoo BC, et al. Clinicopathologic features and prognosis of combined hepatocellular cholangiocarcinoma. American journal of surgery. 2005; 189: 120-5.

7. ALLEN RA, LISA JR. Combined liver cell and bile duct carcinoma. The American journal of pathology. 1949; 25: 647-55.

8. EDMONDSON H, STEINER P. Primary carcinoma of the liver: a study of 100 cases among 48,900 necropsies. Cancer. 1954; 7: 462-503.

9. Bosman FT, Carneiro F, Hruban RH, Theise ND. WHO classification of tumours of the digestive system: World Health Organization. 2010.
10. Nagtegaal ID, Odze RD, Klimstra D, Paradis V, Rugge M, Schirmacher P, et al. The 2019 WHO classification of tumours of the digestive system. Histopathology. 2020; 76: 182-8.

11. Holzner ML, Tabrizian P, Parvin-Nejad FP, Fei K, Gunasekaran G, Rocha C, et al. Resection of Mixed Hepatocellular-Cholangiocarcinoma, Hepatocellular Carcinoma, and Intrahepatic Cholangiocarcinoma: A Western Center Experience. Liver transplantation: official publication of the American Association for the Study of Liver Diseases and the International Liver Transplantation Society. 2020.

12. Lee CH, Hsieh SY, Chang CJ, Lin YJ. Comparison of clinical characteristics of combined hepatocellular-cholangiocarcinoma and other primary liver cancers. Journal of gastroenterology and hepatology. 2013; 28: 122-7.

13. Park HS, Bae JS, Jang KY, Lee JH, Yu HC, Jung JH, et al. Clinicopathologic Study on Combined Hepatocellular Carcinoma and Cholangiocarcinoma: with Emphasis on the Intermediate Cell Morphology. Journal of Korean Medical Science. 2011; 26: 1023.

14. Shibahara J, Hayashi A, Misumi K, Sakamoto Y, Arita J, Hasegawa K, et al. Clinicopathologic Characteristics of Hepatocellular Carcinoma With Reactive Ductule-like Components, a Subset of Liver Cancer Currently Classified as Combined Hepatocellular-Cholangiocarcinoma With Stem-Cell Features, Typical Subtype. The American journal of surgical pathology. 2016; 40: 608-16.

15. Spolverato G, Bagante F, Tsilimigras D, Ejaz A, Cloyd J, Pawlik TM. Management and outcomes among patients with mixed hepatocholangiocellular carcinoma: A population-based analysis. Journal of Surgical Oncology. 2019; 119(3):278-287.

16. Zhao Q, Yu W-L, Lu X-Y, Dong H, Gu Y-J, Sheng X, et al. Combined hepatocellular and cholangiocarcinoma originating from the same clone: a pathomolecular evidence-based study. Cancer Communications. 2016; 35.

17. Bergquist JR, Groeschl RT, Ivanics T, Shubert CR, Habermann EB, Kendrick $\mathrm{ML}$, et al. Mixed hepatocellular and cholangiocarcinoma: a rare tumor with a mix of parent phenotypic characteristics. Hpb. 2016; 18: 886-92.

18. Yoon Y-I, Hwang S, Lee Y-J, Kim K-H, Ahn C-S, Moon D-B, et al. Postresection Outcomes of Combined Hepatocellular Carcinoma-Cholangiocarcinoma, Hepatocellular Carcinoma and Intrahepatic Cholangiocarcinoma. Journal of Gastrointestinal Surgery. 2015; 20: 411-20.

19. Lee JH, Chung GE, Yu S, Hwang S, Kim J, Kim H, et al. Long-term prognosis of combined hepatocellular and cholangiocarcinoma after curative resection comparison with hepatocellular carcinoma and cholangiocarcinoma. Journal of clinical gastroenterology. 2011; 45: 69-75.

20. Brunt E, Aishima S, Clavien P, Fowler K, Goodman Z, Gores G, et al. cHCC-CCA: Consensus terminology for primary liver carcinomas with both hepatocytic and cholangiocytic differentation. Hepatology (Baltimore, Md). 2018; 68: 113-26.

21. Shuqun C, Mengchao W, Han C, Feng S, Jiahe Y, Guanghui D, et al. Tumor thrombus types influence the prognosis of hepatocellular carcinoma with the tumor thrombi in the portal vein. Hepato-gastroenterology. 2007; 54: 499-502.

22. Zhan $\mathrm{Q}$, Shen $\mathrm{BY}$, Deng $\mathrm{XX}$, Zhu ZC, Chen $\mathrm{H}$, Peng $\mathrm{CH}$, et al. Clinical and pathological analysis of 27 patients with combined hepatocellularcholangiocarcinoma in an Asian center. Journal of hepato-biliary-pancreatic sciences. 2012; 19: 361-9.

23. Chantajitr S, Wilasrusmee C, Lertsitichai P, Phromsopha N. Combined hepatocellular and cholangiocarcinoma: clinical features and prognostic study in a Thai population. Journal of Hepato-Biliary-Pancreatic Surgery. 2006; 13: $537-42$.

24. Leoni S, Sansone V, De Lorenzo S, Ielasi L, Tovoli F, Renzulli M, et al. Treatment of Combined Hepatocellular and Cholangiocarcinoma. Cancers. 2020; 12: 794.

25. Bhagat V, Javle M, Yu J, Agrawal A, Gibbs J, Kuvshinoff B, et al. Combined hepatocholangiocarcinoma: case-series and review of literature. International journal of gastrointestinal cancer. 2006; 37: 27-34.

26. Li DB, Si XY, Wang SJ, Zhou Y-M. Long-term outcomes of combined hepatocellular-cholangiocarcinoma after hepatectomy or liver transplantation: A systematic review and meta-analysis. Hepatobiliary \& Pancreatic Diseases International. 2019; 18: 12-8.

27. Peng BG, He Q, Li J, Zhou F. Adjuvant transcatheter arterial chemoembolization improves efficacy of hepatectomy for patients with hepatocellular carcinoma and portal vein tumor thrombus. American journal of surgery. 2009; 198: 313-8.

28. Jiang $\mathrm{J}, \mathrm{Hu} \mathrm{H}$, Liu $\mathrm{R}$, Wang $\mathrm{JH}$, Long $\mathrm{XR}$, Fan $\mathrm{J}$, et al Nomogram for individualized prediction of recurrence after postoperative adjuvant TACE for hepatitis B virus-related hepatocellular carcinoma. Medicine. 2017; 96: e7390.

29. Chu HH, Kim JH, Shim J, Yoon S, Kim P, Alrashidi I. Chemoembolization Plus Radiotherapy Versus Chemoembolization Plus Sorafenib for the Treatment of Hepatocellular Carcinoma Invading the Portal Vein: A Propensity Score Matching Analysis. Cancers. 2020; 12(5):1116.

30. He S, Fan X, Ma H, Xiaerfuhazi $\mathrm{H}$, Rehato A, Feng $\mathrm{I}$, et al. Effect of prophylactic TACE on 5-year survival of patients with hepatocellular carcinoma after hepatectomy. Oncology Letters. 2019.

31. Kim JH, Yoon HK, Ko GY, Gwon D, Jang C, Song H, et al. Nonresectable combined hepatocellular carcinoma and cholangiocarcinoma: analysis of the response and prognostic factors after transcatheter arterial chemoembolization. Radiology. 2010; 255: 270-7.

32. Kassahun WT, Hauss J. Management of combined hepatocellular and cholangiocarcinoma. International Journal of Clinical Practice. 2008; 62: 1271-8. 
33. Kim KH, Lee SG, Park EH, Hwang S, Ahn CS, Moon DB, et al. Surgical Treatments and Prognoses of Patients with Combined Hepatocellular Carcinoma and Cholangiocarcinoma. Annals of Surgical Oncology. 2009; 16: 623-9.

34. Zhang J, Huang Z, Cao L, Zhang Z, Wei Y, Zhang X, et al. Differentiation combined hepatocellular and cholangiocarcinoma from intrahepatic cholangiocarcinoma based on radiomics machine learning. Annals of translational medicine. 2020; 8: 119

35. Liu M, Shi J, Mou T, Wang Y, Wu Z, Shen A. Systematic review of hepatic arterial infusion chemotherapy versus sorafenib in patients with hepatocellular carcinoma with portal vein tumor thrombosis. Journal of gastroenterology and hepatology. 2020; 35: 1277-87.

36. Primrose JN, Fox RP, Palmer DH, Malik HZ, Prasad R, Mirza D, et al. Capecitabine compared with observation in resected biliary tract cancer (BILCAP): a randomised, controlled, multicentre, phase 3 study. The Lancet Oncology. 2019; 20: 663-73.

37. Ma KW, Chok KSH. Importance of surgical margin in the outcomes of hepatocholangiocarcinoma. World journal of hepatology. 2017; 9: 635-41. 